\title{
Novel Synergistic Protective Efficacy of Atovaquone and Diclazuril on Fetal-Maternal Toxoplasmosis
}

\author{
Helieh S. Oz \\ Department of Internal Medicine, University of Kentucky Medical Center, Lexington, KY, USA \\ Email: hoz2@email.uky.edu
}

Received 16 June 2014; revised 15 July 2014; accepted 11 August 2014

Copyright (C) 2014 by author and Scientific Research Publishing Inc.

This work is licensed under the Creative Commons Attribution International License (CC BY). http://creativecommons.org/licenses/by/4.0/

(c) (i) Open Access

\section{Abstract}

Over 1 billion people globally are estimated to be infected with Toxoplasma gondii with severe or unknown consequences and no safe and effective therapies are available against congenital or persistent chronic infection. We propose that atovaquone and diclazuril synergistically protect against fetal-maternal toxoplasmosis. Methods: Programmed pregnant mice were treated with atovaquone and diclazuril monotherapy, or combined (atovaquone + diclazuril) therapy and infected with tachyzoites $(0,300,600)$ and the course of infection was studied. Results: Infected dams with low dose (300) developed moderate toxoplasmosis complications and treatments were similarly effective with minor differences between monotherapies. In contrast, major differences were observed amongst varied treatments during high-dose (600) infection and severe relatedtoxoplasmosis complications as follows. Dams developed hydrothorax, ascities and excess weight gain. Combined therapy $(P<0.01)$ and to a lesser extent diclazuril monotherapy $(P<0.05)$ protected dams from excess weight, hydrothorax, and ascities. Infected dams exhibited splenomegaly, hepatomegaly and severe hepatitis. Combined therapy synergistically normalized pathology $(P<$ 0.001 ) and to a lesser degree monotherapy (diclazuril $P<0.01$, and atovaquone $P<0.05$ ) protected dams from hepatitis and splemomegaly. Additionally, behavioral response to pain stimuli and fetal weight and fetal numbers were significantly preserved in treated dams. Conclusions: This is the first report describing combined atovaquone and diclazuril therapy a) to be safe in pregnancy, b) to exert novel synergistic effects, and c) to protect dams and their nested fetuses against adverse effects of severe toxoplasmosis.

\section{Keywords}

Toxoplasma, Atovaquone, Diclazuril, Synergistic, Protection, Fetal Maternal, Congenital Toxoplasmosis, Pregnancy, Gastroenteritis 


\section{Introduction}

Over 1 billion people globally are estimated to be infected with Toxoplasma gondii, a widespread cause of foodborne disease and congenital toxoplasmosis of which diseases include severe health complications which are endemic particularly in rural areas [1]. Toxoplasma is classified as a Category B human infectious pathogen by the Center for Disease Control (CDC) and National Institute of Health. Acute toxoplasmosis in immunocompetent people is typically symptomless or appears as flu syndrome; however, it leads to demise in immunocompromised patients [2]. Fetal-maternal transmission results in severe complications for the fetus and neonates with potential lifelong adverse consequences [3]-[5]. Congenital toxoplasmosis occurs when the fetus of a seronegative woman or animal becomes infected with organism or a clinically quiescent maternal infection reactivates, typically associated with pregnancy immunosuppression [3].

Toxoplasmosis can manifest as gastrointestinal complications, spontaneous abortion, intrauterine fetal death, preterm delivery, or severe fetal malformations, including ocular, cerebral syndromes, and encephalitis [6]. It is considered as the third most common foodborne cause of hospitalization and death in patients [1] [7] although frequently misdiagnosed or under-diagnosed [8]. From 1,500,000 predicted cases of toxoplasmosis, only small fraction (15\%) is clinically detected each year in USA [9].

Toxoplasma invades the central nervous system and affects the brain development with pathological as well as psycho-behavioral alteration including mental retardation [10]-[12]. Recently, maternal exposure or latent infection has been linked to possible increased risk for autism and schizophrenia, and this theory has received considerable scientific and media attention.

There is no safe and effective (FDA approved) therapy to prevent/eradicate the chronic infection or protect against congenital toxoplasmosis. Therefore, identification of new and highly effective therapeutic agents to prevent and/or treat toxoplasmosis is required to impact this disease. Current therapy for congenital toxoplasmosis relies primarily on spiramycin associated with pyrimethamine-sulfadoxine combined therapy, with the goal of preventing transfer of organism from the actively infected mother to the fetus. However, the adverse effect of treatment with these potentially fetotoxic drugs must be considered [13]-[16]. Pyrimethamine is a pregnancy category $\mathrm{C}$ drug and can cause bone marrow suppression in both mother and inborn. Other treatments have included azithromycin, clarithromycin, spiramycin, atovaquone, dapsone, and cotrimoxazole (trimethoprim sulfamethoxazole) although their efficacy is not proven [17]. Similarly, spiramycin monotherapy can be effective only when administered early in pregnancy and used principally as a preventive measure [16]. More than half (58\%) of the patients received spiramycin retain Toxoplasma DNA in their peripheral blood as they remained infected [15]. In France of 257 live births from sera positive women treated with spiramycin and pyrimethamine-sulfadoxine combined treatment, 66 (24\%) infants were found to be infected [18].

Atovaquone is an FDA approved toxoplasmosis treatment but not for use in congenital toxoplasmosis [13] [19]. Atovaquone is an effective drug against opportunistic disease Pneumocystis pneumonia [20], plasmodial infections [21], and Babesia microti causative of human babesiosis [22] [23].

Another compound, diclazuril, and its related benzeneacetonitriles have long been used in treatment and prevention of poultry and livestock coccidiosis [24] as well as equine protozoal myeloencephalitis (EPM) [25] [26]. Diclazuril is known to be a safe compound at therapeutic dose levels [24]-[26]. Diclazuril principally targets chloroplast derived chlorophyll a-D1 complex which is only present in apicomplexan and not in mammalian organelles [27].

Recently, we have reported the efficacy of atovaquone and diclazuril monotherapy to protect dams against inflammatory and infectious aspects of mild-to-moderate toxoplasmosis [19] [28]. Therapeutic atovaquone and diclazuril combined therapy have not previously been reported nor tested in pregnancy or for toxoplasmosis.

The objectives of this investigation were to evaluate the synergistic efficacy of atovaquone and diclazuril combined therapy against the progression of the maternal fetal toxoplasmosis in our pregnancy model.

\section{Materials and Methods}

\subsection{Ethics}

This research was conducted according to the guidelines and approved by the Institutional Biosaftey Committee (IBC) and the Care and Use of Laboratory Animal Care (IACUC) at University of Kentucky Medical Center. 


\subsection{Toxoplasma gondii Propagation}

Toxoplasma Type II isolates including ME-49 strain are reported predominant in human congenital Toxoplasmosis [29]. For this investigation, Toxoplasma organisms from PTG strain (ME-49, ATCC50841) were originally cloned and propagated by Dr. Daniel Howe of the Maxwell H. Gluck Equine Research Center at the University of Kentucky [19] [28] [30]. Briefly, Tachyzoites were cultured by serial passage in bovine turbinate cells and maintained in minimum essential medium (MEMRS, HyClone Labs, Inc.) supplemented with $4 \%$ fetal clone III (HyClone, Labs, Inc.), Penicillin/streptomycin/fungizone (BioWhittaker, Inc.), and nonessential amino acids solution (HyClone, Labs, Inc.). Upon disruption of the host cell monolayer, extracellular tachyzoites were harvested and purified from host cell debris by filtration through $3.0 \mu \mathrm{m}$ membranes. Tachyzoites were enumerated in a hemocytometer and suspended in phosphate buffer saline (PBS) to the appropriate concentrations for inoculation. All inoculations were administered intraperitoneally (i.p.) in $100 \mu \mathrm{L}$ volume into dams within $1 \mathrm{~h}$ of harvesting to ensure viability.

\subsection{Murine Fetal Toxoplasmosis Model}

Day 1 programmed pregnant (9 weeks old) CD1 mice were purchased from Charles River Lab Inc., Wilmington, MA). Dams were housed individually in microisolator cages in a pathogen free environment and maintained at $22^{\circ} \mathrm{C}$ with a 12:12 hr light-dark cycle at the Maxwell H. Gluck Equine Research Center Laboratory Animal Facility. Animals were fed irradiated rodent chow and sterilized drinking water ad libitum. After 5 days acclimation, dams were weighed and ear punched for appropriate identification. Dams were treated with regimens and on day 8 assigned into 6 - 8 animals per group and injected i.p. with $100 \mu \mathrm{L}$ PBS containing 0, 300, or 600 tachyzoites with $0.5 \mathrm{~mL}$ insulin syringes. Control dams received $100 \mu \mathrm{L}$ sham injection with PBS alone [19]. Animals were monitored daily 3 times for distress, pain, physical appearance, and vaginal discharge to detect abortion or early delivery [19] [31]. The experiment was terminated on gestation day 16 before possible early or premature birth to study the fetal and maternal aspects of the disease.

\subsection{Atovaquone and Diclazuril Treatments}

In order to study safety and efficacy of atovaquone and diclazuril against fetal-maternal toxoplasmosis, animals were divided into groups of 18 - 24 animals. Dams received regimens, atovaquone monotherapy, diclazuril monotherapy, atovaquone + diclazuril combined therapy, or sham incorporated into daily diet as indicated in our previous publications [19] [28]. The control group received sham treatment (inert talcum powder). Treatments were initiated on Day 5 of pregnancy and continued until day 16. On day 8 of pregnancy dams on treatments or sham control arms were further divided into 3 subgroups of 6 - 8 animals and were injected each with 0 (PBS), 300 , or 600 tachyzoites and treatments were continued until dams were euthanatized. Pregnant animals voluntarily consumed their diets with no significant changes in their appearance, food consumption, or weight loss/gain.

\subsection{Sample Collection}

Animals were euthanatized using $\mathrm{CO}_{2}$ inhalation. Immediately their chests were opened and blood from heart collected in microtainers (BD Biosource, Rockville, MD) for hematocrit evaluation. Sera were separated and stored frozen at $-80^{\circ} \mathrm{C}$. The splenic weight and length were recorded. Heart, liver, and uterus were excised and weighed. Colonic contents were removed and colonic length and weigh data measured and flash frozen in liquid nitrogen and stored at $-80^{\circ} \mathrm{C}$ for future studies [32] [33]. Live fetuses were removed from uteri, counted, and weighed and their lengths measured using a digital caliper. All aspects of the investigation were performed according to the guidelines by Institutional Biosafety Committee (IBC) and IACUC at University of Kentucky Medical Center.

\subsection{Histopathological Examination}

A portion of the right lobe from liver tissues of each dam was placed in cassette and fixed with $10 \%$ neutral PBS formalin. The specimens were dehydrated and embedded in paraffin, and tissue sections of $5 \mu \mathrm{m}$ were stained by Hematoxylin Eosin (H\&E) [19] [34]. Each slide was evaluated under Ziess light microscopy. Hepatic lesions were graded on a scale of 0 to $4+$ based on degeneration, inflammation, and necrosis as follows.

(Grade 0)—no detectable lesions, no degeneration, infiltration of inflammatory cells, and normal tissue ap- 
pearance.

(Grade 1) - focal infiltration of inflammatory cells in the tissue and hepatocytes degeneration.

(Grade 2)—mild multifocal infiltration of inflammatory cells, and hepatocytes degeneration.

(Grade 3) - moderate multifocal infiltration of inflammatory cells and hepatocytes degeneration.

(Grade 4)—severe diffuse infiltration of inflammatory cells and necrosis.

\subsubsection{Giemsa Staining}

Giemsa is a delicate polychromatic stain that reveals the fine nuclear detail of Toxoplasma organisms. Giemsa stain contains methylene blue azure basic (MBAB) dyes combined with eosin acidic dyes. The deparaffinized slide sections were stained with the polychromatic Giemsa (40 drops/50 mL distilled water) to stain nuclei of the Toxoplasma organisms and to permit differentiation among the cells. Then, the slides were depreciated in $1 \%$ glacial acetic acid, dehydrated in alcohol and xylene series, and mounted in synthetic resin on slides.

\subsubsection{Immunohistochemical Staining (IHC)}

Anti-Toxoplasma antibody and IHC procedure were kindly provided by Dr. David S. Lindsay at University of West Virginal. Briefly, paraffin-embedded sections were cut, deparaffinized with xylene, rehydrated in alcohol baths, washed in PBS with 0.1\% BSA, quenched endogenous peroxidase activity by incubating in 3\% hydrogen peroxide in methanol for $30 \mathrm{~min}$, and then blocked with rabbit serum (Dako number 1699), 30 min. The sections were incubated with polyclonal Rh anti-Toxoplasma antibody, diluted 1:500 for 90 min, and developed with DAB-chromogen (Dako Carpenteria, CA) for about 5 min until signal developed and subsequently counterstained with hematoxylin then ammonia treated dehydrate stepwise through alcohol, clear with xylene [28].

\subsection{Behavioral Test: Assessment of Pain Related Mechanical Allodynia by Testing Abdominal Withdrawal Threshold}

Abdominal withdrawal responses to mechanical stimuli were quantified with von Frey monofilaments (SemmesWeinstein Anesthesiometer Kit) according to our previous publications with some modification [19] [28]. Briefly, mice were placed into plastic enclosures on the custom-made screen meshed platform. The monofilament range used for this study included 5 different intensities corresponding to (hair diameter) gram force ((4.08) $1.0 \mathrm{~g}$; (3.61) $0.4 \mathrm{~g}$; (3.22) $0.166 \mathrm{~g}$; (2.83) 0.07; (2.36) 0.02 g forces). Testing for mechanical stimulation was performed on the first and the last days of treatment. A single trial consisted of 5 applications of the each filament used once every 6 seconds to allow dam to cease any response and return to an inactive position. Mean values of the percentage of responses of the abdominal withdrawal to each filament (mean withdrawal/5 $\times 100$ ) were used as \% scores for this study. This behavioral test reflected basal level for reflex score and any possible sensory changes observed in the treated mice. A total of 4 dams were tested per each group.

\subsection{Statistical Analysis}

Results are expressed as mean \pm SEM unless otherwise stated. Data were evaluated with ANOVA followed by appropriate post hoc test (Tukey compared all pairs) using GraphPad Instat Version 3 for Windows (Graph-Pad Software, San Diego, CA). Statistical significance was set at $P<0.05$.

\section{Results}

\subsection{Drugs' Safety and Tolerance}

In the initial study, naïve dams were provided diets containing atovaquone, diclazuril, and atovaquone + diclazuril combined therapy, or sham treatment. Dams voluntarily consumed their diets and no adverse effect, such as clinical symptoms and changes in body weight gain rate or appearance were detected compared to those received sham treatment.

\subsection{Drugs' Efficacy}

Next dams were divided into groups and received 1) atovaquone monotherapy, 2) diclazuril monotherapy, 3) atovaquone + diclazuril combined, or 4) sham treatment. Each group was infected with 0, 300, or 600 tachyzoites. Infected dams developed moderate (300) to severe infection (600). Treatments overall were similarly ef- 
fective in groups infected with lower dose (300) as manifested with moderate toxoplasmosis with minor differences between monotherapies. In contrast, there were major differences amongst groups treated with varied regimens and high dose (600) induced infection. Therefore, here we essentially present data from groups infected with the high dose (600) organisms. Sham treated infected dams developed severe toxoplasmosis related inflammation, hydrothorax, ascities, anemia (37 \pm 2 versus controls $44.5 \pm 1.2$ ) and gained excess weight (60\%) compared to controls. Combined therapy and diclazuril monotherapy protected dams from anemia (41.1 \pm 2.4$)$. While, most effective, combined therapy (atovaquone + diclazuril, $P<0.01$ ) synergistically eliminated hydrothorax and ascites and to a lesser extent diclazuril monotherapy $(P<0.05)$ protected dams from excess pathological weight gain (Figure 1(a)).

\subsection{Pain Related Abdominal Response to Stimuli and Therapies}

Infected dams showed a significant pain related abdominal hypersensitivity $(P<0.05)$ to mechanical stimuli as demonstrated with abdominal spasm and withdrawal compared to the uninfected controls. Combined therapy was equally effective as atovaquone monotherapy in preserving the normal abdominal response to stimuli in infected treated dams as compared to infected sham treated animals (Figure 1(b)). In contrast diclazuril monotherapy did not significantly improve behavioral response to pain stimuli in infected dams.

\subsection{Splenomegaly}

Infected dams showed severe splenomegaly (X3 Fold) with loss of germinal structure and massive infiltration of epithelioid cells with significant increases in weight and length of splenic tissues and combined therapy synergistically normalized these anomalies and protected dams from severe infection (Figure 2(a) and Figure 2(b)).

\subsection{Hepatitis}

The major striking synergistic effect of combined therapy with atovaquone + diclazuril was noted in the manifestation of hepatic protection compared to the monotherapy. Hepatic tissues became pale in appearance and enlarged with drastic increases in weight due to severe infection followed by the inflammatory response in sham treated dams (normal versus infected $P<0.001$ ) (Figure 3(a)). Combined therapy synergistically preserved he-

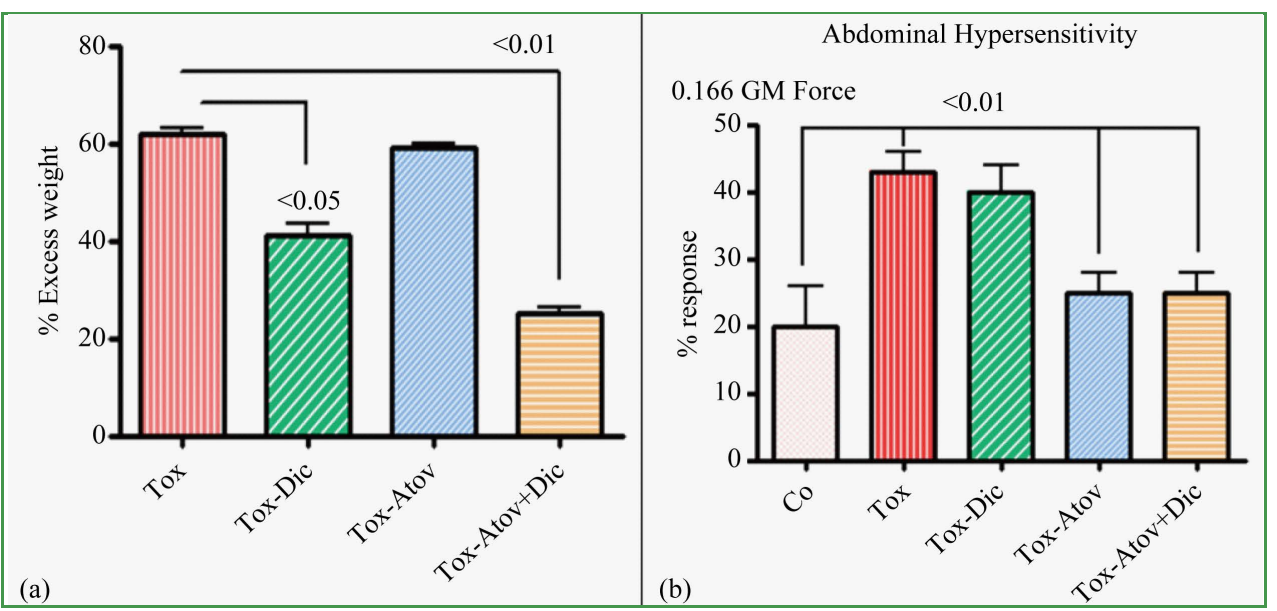

Figure 1. (a) Percent of excess body weight gain. Infected sham treated dams (Tox) gained excess weight $(P<0.001)$ along with inflammation and edema, compared to the uninfected normal controls. Combined (atovaquone + diclazuril) therapy synergistically $(P<0.01)$ and to a lesser extent diclazuril monotherapy $(P<0.05)$ protected dams from excess weight gain $(n=8$ /group). (b) Percent of pain related abdominal response to von Frey mechanical stimuli with 0.166 GM force. Abdominal hypersensitivity significantly increased in infected sham treated dams (Tox) and combined therapy or atovaquone monotherapy was similarly effective and normalized this pain induced behavioral modification in dams. In contrast diclazuril monotherapy had no effect on hypersensitivity to von Frey induced sensation ( $n=4$ /group). 


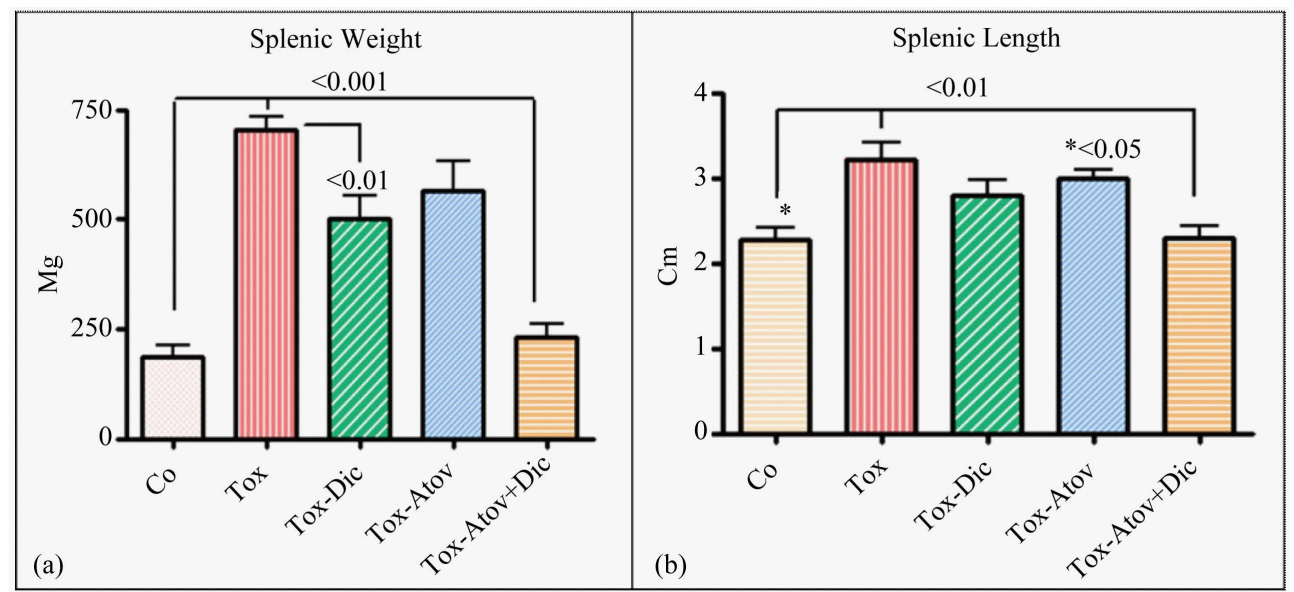

Figure 2. Splenic weight $(P<0.001$ (a)) and length $(P<0.01$ (b)) increased due to inflammatory response. Infected sham treated dams (Tox) and combined therapy (Atov + Dic) synergistically normalized the pathological weight and length increases, while atovaquone or diclazuril monotherapy had a partial but significant therapeutic effect on splenic length or weight ( $n=8$ /group).
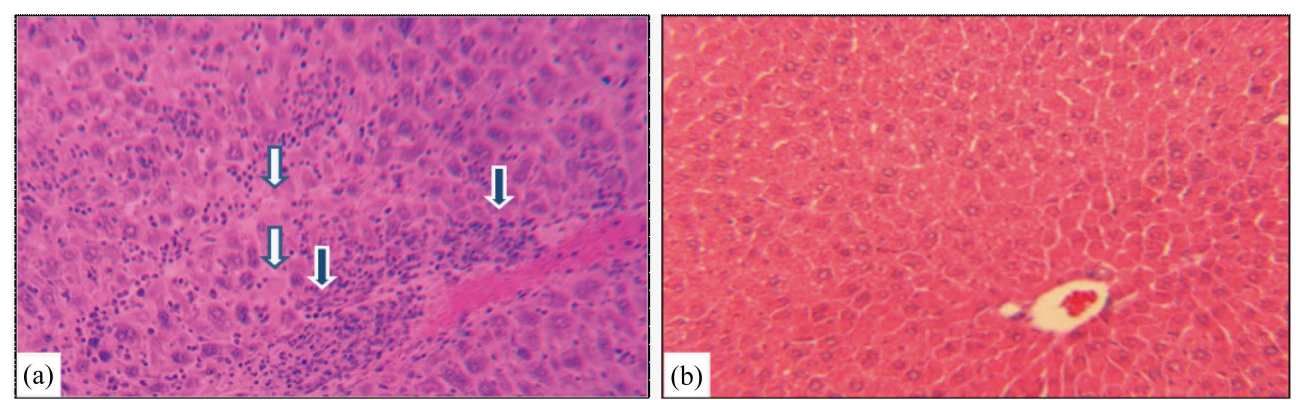

Figure 3. Hepatic section slides representative stained with H\&E from (a) Toxoplasma infected sham treated dam with distorted hepatic architecture, infiltration of mononuclear and polymorphonuclear inflammatory cells (open arrows), degenerated and necrotic hepatocytes (closed arrows). (b) Toxoplasma infected combined atovaquone + diclazuril treated dam with no visible hepatic inflammatory response and injury $(n=8 / g)$.

patic appearance and weight (respectively, sham versus combined therapy $P<0.001$ and versus diclazuril $P<$ 0.01) and microstructural damages (Figure 3(b), Figure 4(a)). Histopathological investigation revealed severe hepatitis with multinucleated dysplastic hepatocytes and giant cell transformation, influx of inflammatory and plasma cells, and hepatic cells necrosis. Accordingly, combined atovaquone +diclazuril therapy synergistically ameliorated hepatic pathological scores superior to monotherapy (respectively, sham versus combined therapy $P$ $<0.001$, versus diclazuril $P<0.01$, and versus atovaquone $P<0.05$ ) (Figure 4(b)).

\subsection{Fetal Health}

Uteri weight significantly increased due to inflammatory cells infiltration and edema in infected dams and partially improved in treated dams which did not reach significant (Table 1). This may be explained due to the increased number of healthy fetuses. Infected dams carried retarded fetuses (normal versus infected, $P<0.001$ ) with sporadic cases of stillbirth or preterm delivery. Combined treatment and atovaquone monotherapy in a similar manner and to a lesser degree diclazuril monotherapy $(P<0.01)$ preserved the number of fetuses, fetal length, fetal weight and protected nested fetuses from retardation and demise (Figure 5).

\section{Discussion}

The global Toxoplasma infectivity ranges from $10 \%$ to over $90 \%$ [35] with estimated incidence rate of congenital toxoplasmosis to be 1.5 cases per 1000 live births [36]. Toxoplasmosis, a "common neglected disease of 


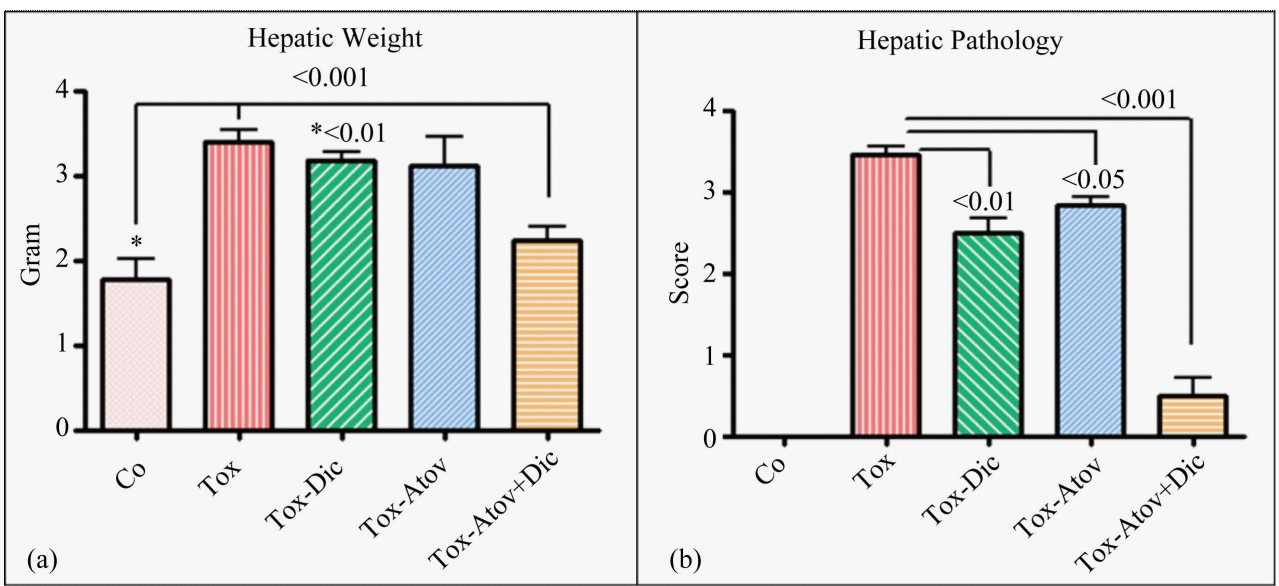

Figure 4. Hepatic weight (a) and hepatic sections stained with H\&E scored from 0 (normal) to 4 (most severe lesions) for pathological findings (b) from controls (Co) and infected sham treated dams (Tox) compared with those with diclazuril (Dic) and atovaquone (Atov) monotherapy or infected combined diclazuril + atovaquone (Dic + Atov) therapy. Hepatic weight (a) and pathological score (b) increased significantly $(P<0.001)$ due to infiltration of inflammatory cells and combined therapy $(P<0.001)$ and diclazuril $(P<0.01)$, respectively, protected the dams from hepatic injuries ( $n=8$ /group).

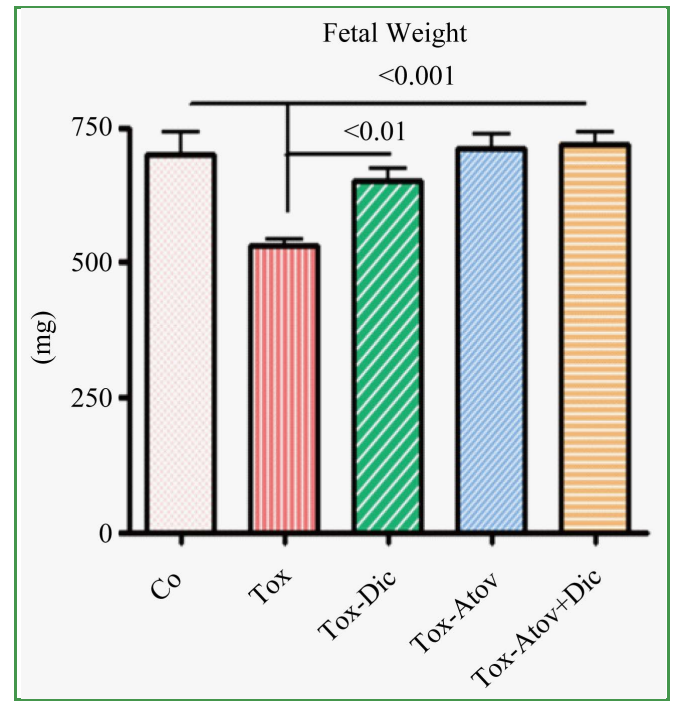

Figure 5. Fetal weight from dams infected with tachyzoites and sham treated compared with atovaquone + diclazuril combined therapy ( $n=8$ /group).

Table 1. Synergistic efficacy of atovaquone and diclazuril combined treatment and monotherapy on Toxoplasma infected dams.

\begin{tabular}{cccccc}
\hline Tissues & Control & Tox & Tox + Dic & Tox + Atov & Tox + Combined \\
\hline Hematocrit (\%) & $44.5 \pm 1.2$ & $37 \pm 1.4^{\mathrm{a}}$ & $41.1 \pm 2.4$ & $34.7 \pm 3.3^{\mathrm{a}}$ & $40.8 \pm 1.5$ \\
Uterus weight (g) & $11.6 \pm 0.5^{\mathrm{a}}$ & $13.9 \pm 0.7^{\mathrm{a}}$ & $11.9 \pm 1.9$ & $12.9 \pm 1$ & $12.3 \pm 1$ \\
\hline
\end{tabular}

Tissues from normal controls (Control), those infected with 600 tachyzoites and treated with sham (Tox), diclazuril monotherapy (Tox + Dic), atovaquone monotherapy (Tox +Atov), or combined atovaquone and diclazuril treated dams (Tox + Combined). Animals were monitored 3 times/day until 16 of pregnancy before termination. Number 8/each group. $P$ value ${ }^{\mathrm{a}}<0.05$.

poverty" (CDC) with higher prevalence, is estimated in socioeconomically disadvantaged populations in the US including woman, African American, Hispanic, and Native American [37]. Several clinical investigations indi- 
cate the importance of toxoplasmosis syndromes and the need for safe and effective therapeutics specifically for fetal congenital toxoplasmosis. Congenital toxoplasmosis occurs by fetal transplacental transmission of organisms during maternal infection or reactivation of chronic infection. Toxoplasma fetal-maternal transmission was first reported in a 3-day-old infant from New York area in 1934 [38] and ever since the worldwide distribution and the significance of congenital toxoplasmosis have been well documented [9] [35] [36].

Toxoplasmosis can lead to severe consequences and death in immunocompromised patients, fetus, and neonates [1]. In healthy appearing "infected individuals", symptoms of infection may be subtle and include diminished IQ and slight adverse behavioral changes [39]. However, significant toxoplasmosis related syndromes occur in fetal and neonatal as well as in immunodeficient subjects. The related syndromes include ocular and central nervous system toxoplasmosis and possibly autistic and schizophrenia-related complications [10]-[12] as infection may involve brain dopamine changes [40] [41]. According to a longitudinal study, women with chronic toxoplasmosis had a higher risk of self-harm [42]. Another cross-sectional study indicated that Toxoplasma seropositives demonstrate increased risk of suicidal violence [43]. Present therapies for toxoplasmosis include pyrimethamine, sulfasalazine, sulfadiazine, and spiramycin, which are not always effective or have major side effects. Pregnant women who seroconvert are generally given spiramycin in order to reduce the risk of transmission to the fetus. However, patients who receive spiramycin retain Toxoplasma DNA in their peripheral blood [15]. In addition, spiramycin is effective only in early pregnancy and not efficacious when organisms have already penetrated the placenta [16].

In a 20-year prospective clinical trial (1985-2005) of infected mothers (666/676) who were treated with spiramycin alone or combined with pyrimethamine-sulfadoxine with live newborn of which, 112 (17\%) confirmed with congenital toxoplasmosis and 26\% later developed chorioretinitis [14]. Likewise, in another report of live birth from infected moms treated with both spiramycin and pyrimethamine-sulfadoxine, the transmission rates of toxoplasmosis were $7 \%$ in the first, $24 \%$ the second and $59 \%$ in the third trimesters, respectively [18].

Because of these inadequacies of available treatments, there is urgent need for more effective chemotherapeutic modalities with less toxicity to encounter fetal and congenital toxoplasmosis as well as recurrent Toxoplasma related complications.

In the current investigation we used combined atovaquone and diclazuril in our fetal-maternal toxoplasmosis model to demonstrate novel synergistic properties protecting pregnant host and fetus from the adverse effects of exposure to Toxoplasma organisms. The combined therapy had superior effects against toxoplasmosis in comparison to atovaquone and diclazuril monotherapy.

Atovaquone (hydroxy-1,4-naphthoquinone) is an FDA approved drug against acute toxoplasmosis and not approved for pregnancy (Figure 6(a) molecular structure). Atovaquone has been proven to be effective drug against opportunistic disease Pneumocystis pneumonia [20], malarial infections [21], and Babesia microti causative of human babesiosis [22] [23] [44]. Atovaquone is used as prophylactic against opportunistic diseases including toxoplasmosis in HIV/AIDS and immunocompetent patients. Atovaquone has been reported to be tolerated better than other available anti-toxoplasmosis therapies and at least as effective [45]. We have shown atovaquone to be effective against some aspects of mild-to-moderate gastrointestinal toxoplasmosis in the pregnancy model [19].

Another compound, diclazuril [4-chlorophenyl [2,6-dichloro-4-(4,5-dihydro-3H-3,5-dioxo-1,2,4-triazin-2-yl) pheny $l$ acetonitrile] is mainly used in food animals' industry to prevent coccidial infection (Figure 6(b) molecular structure). It is also used to treat clinical cases of protozoan S. neurona induced EPM in horses [24]-[26].

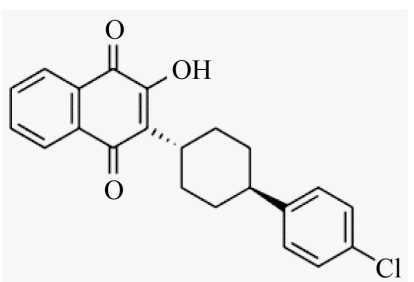

(a)<smiles>N#CC(c1ccc(Cl)cc1)c1c(Cl)cc(-n2ncc(=O)[nH]c2=O)cc1Cl</smiles>

(b)

Figure 6. Molecular structure of atovaquone (a) and diclazuril (b). 
After oral administration, diclazuril is absorbed with steady-state concentrations in plasma and cerebrospinal fluid (CSF) sufficient to interfere with S. neurona proliferation and with 95\% inhibition rate [26]. Diclazuril acts specifically on Toxoplasma protochlorophyllide with traces of chlorophyll a, and binds to the photosynthetic reaction centers [27]. The diclazuril/herbicide-binding site is highly conserved in apicomplexans including Toxoplasma and results in a unique chemotherapeutic sensitivity. Therefore, diclazuril binds the chloroplast epitopes to interact with the D1 protein of the photosynthetic reaction hub of Toxoplasma organelles, without any harmful effect on the host cells. Additionally, diclazuril can downregulate expression of serine/threonine protein phosphatase (EtRACK) in merozoites of Eimeria tenella chicken coccidiosis which results in apoptosis [46]. EtRACK has about 98\% homology with Toxoplasma organisms which demonstrates a predicted mechanism of action for diclazuril against toxoplasmosis. Recently, we reported diclazuril dose dependent effect on moderate gastrointestinal complications in our pregnancy model [28].

In mild-to-moderate infection, diclazuril monotherapy and to a lesser extent atovaquone monotherapy suppressed the Toxoplasma infection [19] [28]. However in severe cases, atovaquone and diclazuril combined therapy was superior to atovaquone or diclazuril monotherapy in synergistically normalizing or ameliorating toxoplasmosis symptoms. These included the improvement of pathologic excess weight gain, ascites, hydrothorax, and severity of splenomegaly. The major differences were noted when dams became infected with high dose of tachyzoites (600) with the manifestation of the hepatic disease. Combined atovaquone and diclazuril exhibited a strong synergistic effect $(P<0.001)$ and protected dams from severe inflammatory infection, while diclazuril monotherapy $(P<0.01)$ or atovaquone monotherapy $(P<0.5)$ only partially protected dams from inflammatory hepatocellular injuries. Additionally, the number of fetuses, fetal length, and weight were significantly preserved in combined therapy. This study demonstrated evidence that atovaquone and diclazuril combined therappy exerts significant synergistic effects in normalization or reduction of the severe pathogenesis and clinical symptoms in maternal and fetal toxoplasmosis with minimum risk to the dams and fetuses.

\section{Conclusion}

This is the first report to establish proof of the principle that a) atovaquone and diclazuril combined therapy exerts novel synergistic efficacies in infectious and inflammatory disease, b) it is safe in pregnancy, and c) it synergistically protects dams and their nested fetuses against severe Toxoplasma infection and adverse toxoplasmosis-related inflammatory complications in pregnancy with no detected side effects. These findings warrant future trials of the combined atovaquone and diclazuril as novel synergistic prophylactic and therapeutic approach to fetal maternal toxoplasmosis.

\section{Conflict of Interests}

The author declares no conflict of interests.

\section{Acknowledgements}

Tachyzoites were provided by Dr. Daniel Howe. Dr. Thomas Tobin from Maxwell H. Gluck Equine Center, College of Agriculture, University of KY, provided a portion of funding from Kentucky Science and Technology KSTC 721-RFP-006 and the concept of diclazuril in congenital toxoplasmosis [28]. Dr. David S. Lindsay kindly provided anti-mouse Toxoplasma specific antibody for immunohistochemical staining. Felicia Kost assisted with animal handling and Dana Napier with preparation of IHC and Giemsa staining. This investigation was supported by the Grant from National Institutes of Health NIH-DE019177 (Dr. Helieh S. Oz). University of Kentucky invention property Invention Disclosure is INV11/1773.

\section{References}

[1] Hoffmann, S., Batz, B.M. and Morris Jr., J.G. (2012) Annual Cost of Illness and Quality-Adjusted Life Year Losses in the United States Due to 14 Foodborne Pathogens. Journal of Food Protection, 75, 1292-1302. http://dx.doi.org/10.4315/0362-028X.JFP-11-417

[2] Dubey, J.P. and Jones, J.L. (2008) Toxoplasma gondii Infection in Humans and Animals in the United States. International Journal for Parasitology, 38, 1257-1278. http://dx.doi.org/10.1016/j.ijpara.2008.03.007

[3] Kieffer, F. and Wallon, M. (2013) Congenital Toxoplasmosis. Handbook of Clinical Neurology, 112, 1099-1101. 
http://dx.doi.org/10.1016/B978-0-444-52910-7.00028-3

[4] Olariu, T.R., Remington, J.S., McLeod, R., Alam, A. and Montoya, J.G. (2011) Severe Congenital Toxoplasmosis in the United States: Clinical and Serologic Findings in Untreated Infants. Pediatric Infectious Disease Journal, 30, 10561061. http://dx.doi.org/10.1097/INF.0b013e3182343096

[5] Remington, J.S., Thulliez, P. and Montoya, J.G. (2004) Recent Developments for Diagnosis of Toxoplasmosis. Journal of Clinical Microbiology, 42, 941-945. http://dx.doi.org/10.1128/JCM.42.3.941-945.2004

[6] Wong, S.-Y. and Remington, J.S. (1994) Toxoplasmosis in Pregnancy. Clinical Infectious Diseases, 18, $853-861$. http://dx.doi.org/10.1093/clinids/18.6.853

[7] Mead, P.S., Slutsker, L., Dietz, V., et al. (1999) Food-Related Illness and Death in the United States. Emerging Infectious Diseases, 5, 607-625. http://dx.doi.org/10.3201/eid0505.990502

[8] Pereira, K.S., Franco, R.M.B. and Leal, D.A.G. (2010) Transmission of Toxoplasmosis (Toxoplasma gondii) by Foods. Advances in Food and Nutrition Research, 60, 1043-4526. http://dx.doi.org/10.1016/S1043-4526(10)60001-0

[9] Jones, J.L., Kruszon-Moran, D., Wilson, M., McQuillan, G., Navin, T. and McAuley, J.B. (2001) Toxoplasma gondii Infection in the United States: Seroprevalence and Risk Factors. The American Journal of Epidemiology, 154, 357-365. http://dx.doi.org/10.1093/aje/154.4.357

[10] Brown, A.S., Schaefer, C.A., Quesenberry Jr., C.P., Liu, L., Babulas, V.P. and Susser, E.S. (2005) Maternal Exposure to Toxoplasmosis and Risk of Schizophrenia in Adult Offspring. The American Journal of Psychiatry, 162, 767-773. http://dx.doi.org/10.1176/appi.ajp.162.4.767

[11] Bachmann, S., Schroder, J., Bottmer, C., Torrey, E.F. and Yolken, R.H. (2005) Psychopathology in First-Episode Schizophrenia and Antibodies to Toxoplasma gondii. Psychopathology, 38, 87-90. http://dx.doi.org/10.1159/000085349

[12] Wang, H.L., Wang, G.H., Li, Q.Y., Shu, C., Jiang, M.S. and Guo, Y. (2006) Prevalence of Toxoplasma Infection in First-Episode Schizophrenia and Comparison between Toxoplasma-Seropositive and Toxoplasma-Seronegative Schizophrenia. Acta Psychiatrica Scandinavica, 114, 40-48. http://dx.doi.org/10.1111/j.1600-0447.2006.00780.x

[13] Cortina-Borja, M., Tan, H.K., Wallon, M., Paul, M., Prusa, A., Buffolano, W., et al. (2010) Prenatal Treatment for Serious Neurological Sequelae of Congenital Toxoplasmosis: An Observational Prospective Cohort Study. PLoS Medicine, 7, Article ID: e1000351. http://dx.doi.org/10.1371/journal.pmed.1000351

[14] Berrebi, A., Assouline, C., Bessieres, M.H., Lathière, M., Cassaing, S., Minville, V. and Ayoubi, J.M. (2010) LongTerm Outcome of Children with Congenital Toxoplasmosis. The American Journal of Obstetrics and Gynecology, 203, 552.e1-552.e6. http://dx.doi.org/10.1016/j.ajog.2010.06.002

[15] Habib, F.A. (2008) Post-Treatment Assessment of Acute Toxoplasma Infection during Pregnancy. Journal of Obstetrics and Gynaecology, 28, 593-595. http://dx.doi.org/10.1080/01443610802344332

[16] Julliac, B., Theophile, H., Begorre, M., Richez, B. and Haramburu, F. (2010) Side Effects of Spiramycin Masquerading as Local Anesthetic Toxicity during Labor Epidural Analgesia. International Journal of Obstetric Anesthesia, 19, 331332. http://dx.doi.org/10.1016/j.ijoa.2010.03.002

[17] Petersen, E. and Schmidt, D.R. (2003) Sulfadiazine and Pyrimethamine in the Postnatal Treatment of Congenital Toxoplasmosis: What Are the Options? Expert Review of Anti-Infective Therapy, 1, 175-182. http://dx.doi.org/10.1586/14787210.1.1.175

[18] Bessieres, M.H., Berrebi, A., Cassaing, S., Fillaux, J., Cambus, J.P., Berry, A., Assouline, C., Ayoubi, J.M. and Magnaval, J.F. (2009) Diagnosis of Congenital Toxoplasmosis: Prenatal and Neonatal Evaluation of Methods Used in Toulouse University Hospital and Incidence of Congenital Toxoplasmosis. Memorias do Instituto Oswaldo Cruz, 104, 389-392. http://dx.doi.org/10.1590/S0074-02762009000200038

[19] Oz, H.S. and Tobin, T. (2012) Atovaquone Ameliorates Gastrointestinal Toxoplasmosis Complications in a Pregnancy Model. Medical Science Monitor, 18, 337-345. http://dx.doi.org/10.12659/MSM.883342

[20] Oz, H.S., Hughes, W.T. and Rehg, J.E. (1999) Rat Model for Dual Opportunistic Pathogen Prophylaxis: Cryptosporidium Parvum and Pneumocystis carinii. Laboratory Animal Science, 49, 331-334.

[21] Hudson, A.T., Dickins, M., Ginger, C.D., Gutteridge, W.E., Holdich, T., Hutchinson, D.B., Pudney, M., Randall, A.W. and Latter, V.S. (1991) 566C80: A Potent Broad Spectrum Anti-Infective Agent with Activity against Malaria and Opportunistic Infections in AIDS Patients. Drugs under Experimental and Clinical Research, 17, 427-435.

[22] Hughes, W.T. and Oz, H.S. (1995) Successful Prevention and Treatment of Babesiosis with Atovaquone. Journal of Infectious Diseases, 172, 1042-1046. http://dx.doi.org/10.1093/infdis/172.4.1042

[23] Oz, H.S. and Westlund, H.K. (2012) Human Babesiosis: An Emerging Transfusion Dilemma. International Journal of Hepatology, 2012, Article ID: 431761. http://dx.doi.org/10.1155/2012/431761

[24] Assis, R.C.L., Luns, F.D., Beletti, M.E., Assis, R.L., Nasser, N.M., Faria, E.S.M. and Cury, M.C. (2010) Histomor- 
phometry and Macroscopic Intestinal Lesions in Broilers Infected with Eimeria acervulina. Veterinary Parasitology, 168, 185-189. http://dx.doi.org/10.1016/j.vetpar.2009.11.017

[25] Granstrom, D.E. and Tobin, T. (1999) Treatment of EPM Formulations and Methods to Treat and Prevent Equine Protozoal Myeloencephalitis. US Patent No. 5883095.

[26] Dirikolu, L., Lehner, F., Nattrass, C., et al. (1999) Diclazuril in the Horse: Its Identification and Detection and Preliminary Pharmacokinetics. Journal of Veterinary Pharmacology and Therapeutics, 22, 374-379. http://dx.doi.org/10.1046/j.1365-2885.1999.00232.x

[27] Hackstein, J.H.P., Mackenstedt, U., Mehlhorn, H., Meijerink, J.P.P., Schubert, H. and Leunissen, J.A.M. (1995) Parasitic Apicomplexans Harbor a Chlorophyll a-D1 Complex, the Potential Target for Therapeutic Triazines. Parasitology Research, 81, 207-216.

[28] Oz, H.S. and Tobin, T. (2014) Diclazuril Protects against Maternal Gastrointestinal Syndrome and Congenital Toxoplasmosis. International Journal of Clinical Medicine, 5, 93-101. http://dx.doi.org/10.4236/ijcm.2014.53017

[29] Ajzenberg, D., Cogne, N., Paris, L., Bessières, M.H., Thulliez, P., Filisetti, D., Pelloux, H., Marty, P. and Dardé, M.L. (2002) Genotype of Toxoplasma gondii Isolates Associated with Human Congenital Toxoplasmosis, and Correlation with Clinical Findings. Journal of Infectious Diseases, 186, 684-689. http://dx.doi.org/10.1086/342663

[30] Howe, D.K., Honore, S., Derouin, F. and Sibley, L.D. (1997) Determination of Genotypes of Toxoplasma gondii Strains Isolated from Patients with Toxoplasmosis. Journal of Clinical Microbiology, 35, 1411-1414.

[31] Oz, H.S., Ebersole, J.L. and de Villiers, W.J.S. (2011) The Macrophage Pattern Recognition Scavenger Receptors SR-A and CD36 Protect against Microbial Induced Pregnancy Loss. Inflammation Research, 60, 93-97. http://dx.doi.org/10.1007/s00011-010-0241-1

[32] Oz, H.S., Chen, T.S. and Nagasawa, H. (2007) Comparative Efficacies of 2 Cysteine Prodrugs and a Glutathione Delivery Agent in a Colitis Model. Translational Research, 150, 122-129. http://dx.doi.org/10.1016/j.trsl.2006.12.010

[33] Oz, H.S., Chen, T. and de Villiers, W.J.S. (2013) Green Tea Polyphenols and Sulfasalazine Have Parallel Anti-Inflammatory Properties in Colitis Models. Frontiers in Immunology, 132, 1-103. http://dx.doi.org/10.3389/fimmu.2013.00132

[34] Oz, H.S., Im, H.J., Chen, T.S., de Villiers, W.J.S. and McClain, C.J. (2006) Glutathione-Enhancing Agents Protect against Steatohepatitis in a Dietary Model. Journal of Biochemical and Molecular Toxicology, 20, 39-47. http://dx.doi.org/10.1002/jbt.20109

[35] Pappas, G., Roussos, N. and Falagas, M.E. (2009) Toxoplasmosis Snapshots: Global Status of Toxoplasma gondii Seroprevalence and Implications for Pregnancy and Congenital Toxoplasmosis. International Journal for Parasitology, 39, 1385-1394. http://dx.doi.org/10.1016/j.ijpara.2009.04.003

[36] Hotez, P.J. (2008) Neglected Infections of Poverty in the United States of America. PLoS Neglected Tropical Diseases, 2, Article ID: e256, 1-11.

[37] Torgerson, R.P. and Mastroiacovo, P. (2013) The Global Burden of Congenital Toxoplasmosis: A Systematic Review. Bulletin of the World Health Organization, 91, 501-508. http://dx.doi.org/10.2471/BLT.12.111732

[38] Wolf, A., Cowen, D. and Paige, B. (1939) Human Toxoplasmosis: Occurrence in Infants as an Encephalomyelitis Verification by Transmission to Animals. Science, 89, 226-227. http://dx.doi.org/10.1126/science.89.2306.226

[39] Flegr, J., Preiss, M., Klose, J., Havlicek, J., Vitakova, M. and Kodym, P. (2003) Decreased Level of Psychobiological Factor Novelty Seeking and Lower Intelligence in Men Latently Infected with the Protozoan Parasite Toxoplasma gondii Dopamine, a Missing Link between Schizophrenia and Toxoplasmosis? Biological Psychology, 63, 253-268. http://dx.doi.org/10.1016/S0301-0511(03)00075-9

[40] Fekadu, A., Shibre, T. and Cleare, A.J. (2010) Toxoplasmosis as a Cause for Behavior Disorders: Overview of Evidence and Mechanisms. Folia Parasitologica, 57, 105-113.

[41] Torrey, E.F., Bartko, J.J., Lun, Z.R. and Yolken, R.H. (2007) Antibodies to Toxoplasma gondii in Patients with Schizophrenia: A Meta-Analysis. Schizophrenia Bulletin, 33, 729-736. http://dx.doi.org/10.1093/schbul/sbl050

[42] Pedersen, M.G., Mortensen, P.B., Norgaard-Pedersen, B. and Postolache, T.T. (2012) Toxoplasma gondii Infection and Self-Directed Violence in Mothers. JAMA Psychiatry, 69, 1123-1130. http://dx.doi.org/10.1001/archgenpsychiatry.2012.668

[43] Zhang, Y., Traskman-Bendz, L., Janelidze, S., Langenberg, P., Saleh, A., Constantine, N., et al. (2012) Toxoplasma gondii Immunoglobulin G Antibodies and Nonfatal Suicidal Self Directed Violence. The Journal Clinical Psychiatry, 73, 1069-1076. http://dx.doi.org/10.4088/JCP.11m07532

[44] Pearson, P.A., Piracha, A.R., Sen, H.A. and Jaffe, G.J. (1999) Atovaquone for the Treatment of Toxoplasma Retinochoroiditis in Immunocompetent Patients. Ophthalmology, 106, 148-153. 
http://dx.doi.org/10.1016/S0161-6420(99)90021-0

[45] Hughes, W. and Oz, H.S. (1997) Successful Prevention and Treatment of Babesiosis with Atovaquone. In: Keusch, G., Ed., The Best of Infectious Diseases, Mosby-Year Book Inc., Saint Louis, 194-196.

[46] Zhou, B.H., Wang, H.W., Zhao, Z.S., Liu, M., Yan, W.C., Zhao, J., Zhang, Z. and Xue, F.Q. (2013) A Novel Serine/ Threonine Protein Phosphatase Type 5 from Second Generation Merozoite of Eimeria tenella Is Associated with Diclazuril-Induced Apoptosis. Parasitology Research, 112, 1771-1780. http://dx.doi.org/10.1007/s00436-013-3336-0

\section{Abbreviations}

BSA: Bovine Serum Albumin

CDC: Center for Disease Control

CNS: Central Nervous System

EPM: Equine Protozoal Myeloencephalitis

FDA: Food and Drug Administration

H\&E: Hematoxylin Eosin

IACUC: Institutional Animal Care Use Committee

IBC: Institutional Biosaftey Committee

IHC: Immunohistochemical Staining

i.p.: Intraperitoneally

n: Number of Animals/Group

MEM: Minimum Essential Medium

PBS: Phosphate Buffer Saline

S. neurona: Sarcocystis neurona

Tox: Toxoplasma gondii 
Scientific Research Publishing (SCIRP) is one of the largest Open Access journal publishers. It is currently publishing more than 200 open access, online, peer-reviewed journals covering a wide range of academic disciplines. SCIRP serves the worldwide academic communities and contributes to the progress and application of science with its publication.

Other selected journals from SCIRP are listed as below. Submit your manuscript to us via either submit@scirp.org or Online Submission Portal.
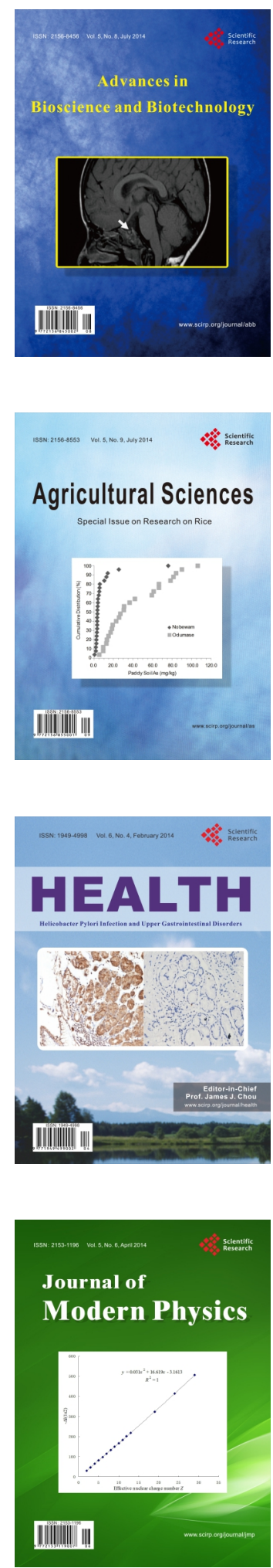
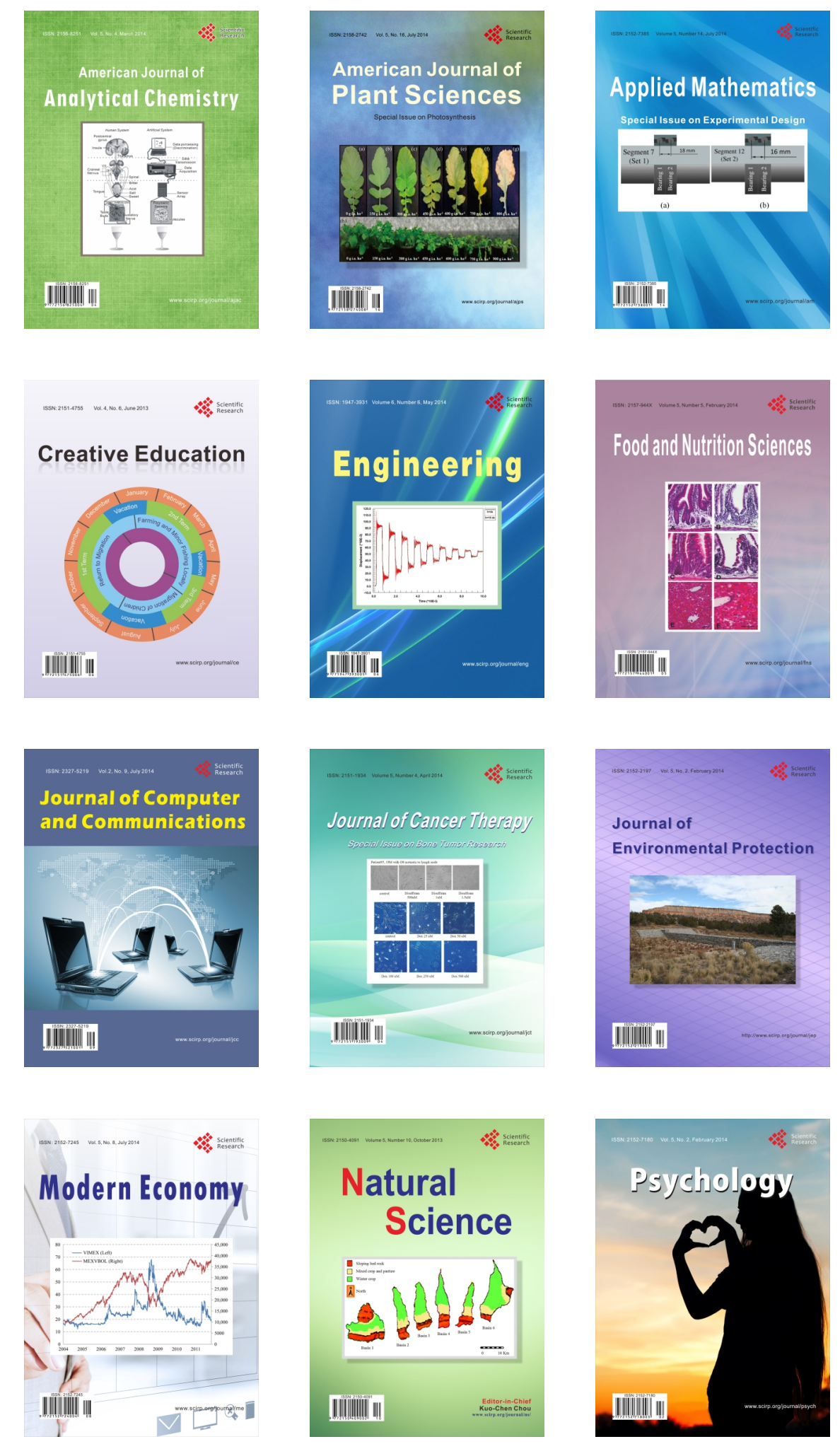